\title{
Fuzzifying Telecommunication Network Features Basing PSO-TE Algorithm
}

\author{
Walaa Elsanabary ${ }^{1, *}$, Mona Gamal $^{2}$, Ahmed Abou El-Fetouh $^{3}$ \\ and Nashaat Elkhameesy ${ }^{4}$ \\ ${ }^{1}$ Assistant Lecturer, Information System Department, Sadat Academy, Egypt \\ ${ }^{2}$ Lecturer, Information System Department, Faculty of Computers \\ And Information, KFS University, Egypt \\ ${ }^{3}$ Professor, Information System Department, Faculty of Computers and \\ Information Sciences, Mansoura University, Egypt \\ ${ }^{4}$ Professor, Information System Department, Sadat Academy, Egypt \\ welsanabary@yahoo.com,Mona2004egypt@yahoo.com, \\ abou_elfetouh@yahoo.com,wessasalsol@gmail.com
}

\begin{abstract}
Planning Telecommunication Access Network (TAN) infrastructure is a real time problem that suffers from uncertainty and ambiguity. Fuzzy system is a discipline that proved its capability to deal with vague problems. Converting traditional data to suit fuzzy system calculations is called fuzzification process which is a crucial step affecting the whole system accuracy? Generating appropriate membership function for fuzzy variables is one of the most challenging issues in fuzzy systems design. This paper proposes a solution to generate membership function automatically. An integrated hybrid model (PSO-TE) is introduced which benefit the Particle Swarm Optimization (PSO) and the information theory measures (entropy and mutual information) as the fitness function to adjust particles (membership function parameters). The proposed algorithm is tested using realistic planning information to show its effectiveness and efficiency. During the comparison between the PSO-TE and the fuzzy C-mean algorithm, the proposed model proved its ability to produce stable membership function for the telecommunication data barriers.
\end{abstract}

Keywords: Particle Swarm Optimization (PSO), Total Entropy (TE), Entropy Function, Mutual Information, Geographic Information System (GIS), Telecommunication Access Network (TAN) Infrastructure

\section{Introduction}

Uncertainty is a typical issue in handling many real time situations. Telecommunication networks utilize the uncertainty analysis in several problems. Planning network infrastructure [8] is an emergent field that decreases cost effects and increases network efficiency. This planning is concerned with network operators and service providers in a time of globalization and huge competition. The current telecommunication market needs flexible and dynamic network planning methodologies for evolving network architectures to meet telecommunications companies and customer requirements.

Fuzzy system[7] is interested in uncertainty analysis of data and inferring knowledge from fuzzy data. The main obstacle is converting traditional data variables into crisp fuzzy data. The fuzzifiaction process turns concrete data variable into a number of fuzzy

${ }^{*}$ Corresponding Author 
subsets or labels along with their membership degrees defining the new fuzzy variable. Losing information of variables after the fuzzification process is a huge problem that needs feasible and efficient solutions.

Applying fuzzy concepts to the planning process for establishment of the Telecommunication Access Network (TAN) Infrastructure would increase the flexibility of network analysis as a whole. The accurate and flexible analysis of providing network services improves the quality of services and reduces capital expenses (CAPEX).

Although, the network concrete data of these variables support the traditional process of site selection, they are inefficient in uncertainty decision situations. These variables should be fuzzified to cope up with the fuzzy systems problems. Generating the membership function [9] of variable subsets is the main issue. The membership truth degrees of different network barriers should facilitate the fuzzy inference process from the network data. Fuzzy inference [7] is a perfect alternative for human experts as linguistic knowledge to solve the fuzzified network site selection problem which could be a future research interest.

In this paper, an integrated hybrid model for generating membership functions for the network data variables is introduced. The hybrid model utilizes the Particle Swarm Optimization used as a meta-heuristic search mechanism and the information theory measures (entropy and mutual information) as the fitness function to adjust particles (membership function parameters) automatically. After convergence, particles achieve the typical result; PSO will upgrade the estimation of parameters to make the whole new fuzzy membership function. The generated membership functions of the telecommunication barriers would be further utilized in making fuzzy decisions in several uncertainty analysis steps.

The rest of this paper is organized as follows: Section II represents a survey in literature for solving the fuzzification process. Section III sets the preliminaries such as particle swarm optimization (PSO), entropy and mutual information. Section IV gives an over view of the input data which used to generate a new membership function, the whole system and its modules. Then the credibility value of the whole system is calculated. Experimental results and conclusion will appear in Sections V and VI respectively.

\section{Literature Reviews}

The fuzzification process is the membership function design for the fuzzy variables, hence it is considered the initialization phase in fuzzy systems. Transforming traditional data into fuzzy data is a critical step in many applications of fuzzy systems. This transformation process is called membership function generation. Many researchers are interested in designing accurate membership functions. Moreover, evaluating and improving the accuracy of any generated model needs to choose suitable fuzzy sets or fuzzy membership functions Wan-I Lee [22] utilize automating membership function to introduce an integrated approach based on genetic fuzzy systems (GFS) and artificial neural networks (ANN) for constructing a stock price forecasting expert system.

Masoud Makrehchi, [17] introduced genetic algorithm optimization and information theory measures for automatic generation of fuzzy membership functions which can be utilized in learning strategies to generate generic membership function for generic data. K.K. Ang and C. Quek generated fuzzy membership functions by Supervised Pseudo Self-Evolving Cerebellar (SPSEC) algorithm which are linguistically interpretable and possessed decision boundary that is equivalent to the probability distributions estimated using the Parzen Window that employed unvaried Gaussian kernel and normal optimal smoothing without relying on any user-defined parameters[10]. A novel TakagiSugeno (TS) fuzzy model with sigmoid-based Membership Functions(MFs). The MFs used in approximation of a nonlinear function introduce first-order and high-order TS fuzzy models. The comparison results demonstrate the relative high accuracy and low 
redundancy of the suggested TS-SBMF fuzzy model [1]. Generating new fuzzy membership function for Fuzzy Support Vector Machine (FSVM)are introduced using combination of three variables: distance, similarity, and depth of data. This method heads for producing high membership degree and it gives effect to the form of decision surface.[15].

Grzegorz Nieradka, Bohdan Butkiewicz [5] introduced new method in determining membership function in the field of image processing based on fuzzy entropy and fuzzy index. Then this research using the optimization algorithm PSO to find the set of optimal parameters. Another research that presents how to control the water levels of a nonlinear water tank system by using PSO to automatically adjust the MFs of a Mamadani-type fuzzy controller in performing tracking control [6].

Another research which care about the adjustment of membership functions via the Particle Filtering, called the PFMFA. It generates some initial random samples and starts to compute the membership function's parameters recursively which is more robust against nonlinearity compared with the Extended Kalman Filter Membership Function Adjustment (EKFMFA)[9]. W. Liao introduce a procedure for Generating Interval Type-2 Membership Functions from Data which called extended $\pi$. This procedure has been shown to cover triangular, trapezoidal, $\mathrm{s}, \mathrm{z}$, and $\pi$ interval type- 2 membership functions as special cases [21].

Other researchers care about generating appropriate membership functions using Particle Swarm Optimization (PSO) technique. One of them introduces a Fuzzy PSO to adjust fuzzy membership function and improve the performance result in terms of accurate estimation[12] . Another research proposed algorithm deal with the problem of quantitative association Rule Mining ARM, the combination of S and Z fuzzy shapes, which is combined with the Particle Swarm Optimization (PSO) to generate appropriate membership[4].

\section{Preliminaries}

\subsection{Representation of Membership Function}

The fuzzy membership function is a main idea in outlining fuzzy systems (FS).A membership function (MF) [18][2][17] is a bend that defines how each point in the input space is mapped to a membership value (or level of membership) between 0 and 1.Different types of membership function have been already proposed such as Triangular, Trapezoidal, Gaussian, Generalized bell, $\pi$-Shaped and S-Shaped Membership Functions. But most popular MF's are: trapezoidal and triangular. Figure 1 shows an example on trapezoidal and triangular MFs.
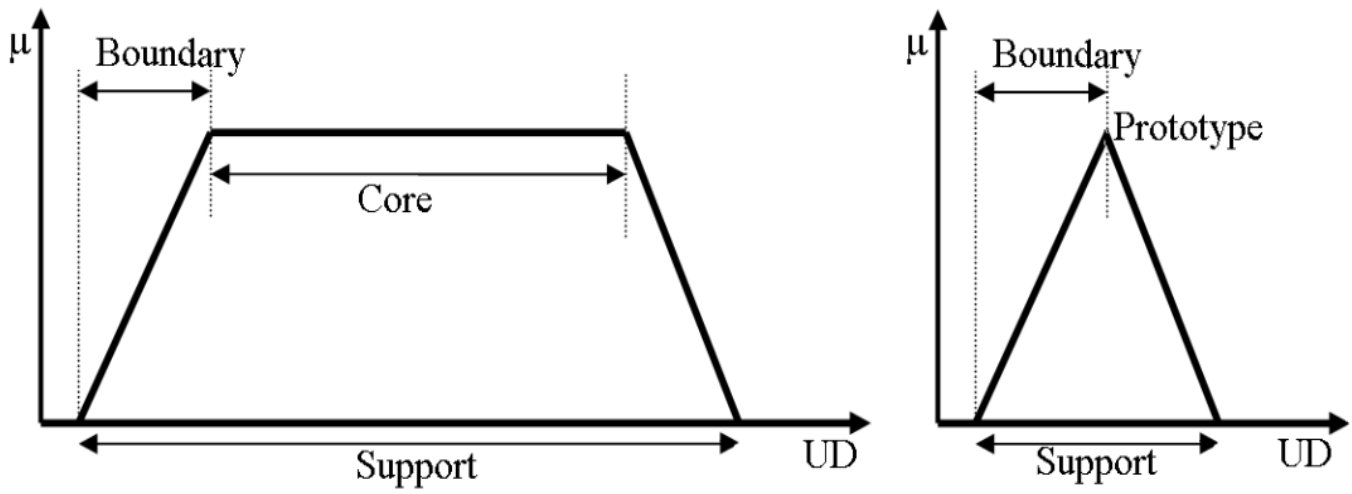

Figure 1. Parameters of MF in Two Most Popular MF's: Trapezoidal and Triangular 


\subsection{Particle Swarm Optimization}

The Particle Swarm Optimization Algorithm (PSO) is a population based improvement strategy that finds the optimum arrangement utilizing a population of particles. In PSO, a swarm is the answer of the problem in the data space. PSO is principally generated through simulation of bird flocking [12].

The fundamental algorithm of PSO comprises three basic phases which are generating particles positions and velocities, velocities adjustment and position adjustment [12][19].

- The positions, xid and velocity, pid of the first swarm of particles are randomly created. The positions and velocity are given in a vector design for the ith particle at time t.

- Upgrade velocities of all particles at time $t+1$ using the current and global best particles objective or fitness values at time t. The fitness value of a particle determines which particle has the best global value in the swarm, pgd, and determines the optimum position of each particle over time, pid(i.e. in current and all previous moves).

- Thevelocity upgrade formula uses these two pieces of information (pgd and pid) for each particle in the swarm along with the effect of current motion, vid(t), to provide a search direction, $\operatorname{vid}(t+1)$, for the next iteration.

\section{Particles Velocity}

$$
v_{i d}(t+1)=w * v_{i d}(t)+c 1 * \text { rand } *\left(p_{i d}-x_{i d}\right)+c 2 * \text { rand } *\left(p_{g 1}-x_{i d}\right)
$$

Update the current position of the particle to obtain its next position:

\section{Particles Position}

$$
x_{i}(t+1)=x_{i}(t)+v_{i}(t+1)
$$

\section{Where}

$\mathrm{c} 1$ and $\mathrm{c} 2$ are positive constants which are random numbers within the range [0,1],

$P_{g l}$ is the global best position,

$p_{i d}$ is the current best position of the particle $\mathrm{i}$, rand is a random value generated in the interval range $[0,1]$, $\mathrm{w}$ is the inertia weight.

\subsection{Entropy and Mutual Information}

The entropy [Ma Jian, Sun Zengqi, 2011] of an arbitrary variables a function which endeavors to characterize the "unpredictability" of an arbitrary variable. Consider an arbitrary variable $X$ takes on values in a set $X=\{x 1, x 2, \ldots, x n\}$, and is defined by a probability distribution $\mathrm{P}(\mathrm{X})$, then the entropy of the random variable can be represented as in equation No 3:

$$
H_{x}=-\sum_{i=1}^{n} P\left(x_{i}\right) \log P\left(x_{i}\right)
$$

Joint entropy of a pair of two discrete random variables $\mathrm{X}$ and $\mathrm{Y}$ is more important to calculate mutual information. Joint entropy can be calculated by equation $\mathrm{NO} 4$ :

$$
H(X, Y)=-\Sigma_{x \in X} \sum_{y \in Y} p(x, y) \log p(x, y)
$$

Mutual Information is an amount that measures a relationship between two arbitrary factors that are examined at the same time. Specifically, it quantifies how much 
information is communicated, on average, in one random variable about another. The mutual information $I(x ; y)$ between the random variables $\mathrm{X}$ and $\mathrm{Y}$ is given by:

$$
I(x ; y)=\sum_{x y} p(x, y) \log \frac{p(x y)}{p(x) p(y)}
$$

\section{The Proposed PSO-TE (Particle Swarm Optimization basing Total Entropy)}

The proposed PSO-TE integrated hybrid model utilizes the meta-heuristic search algorithm PSO and the TE (total entropy function) to find the best parameters to draw membership function for the network fuzzy variable. The proposed model searches the data space for feasible decision variables (membership function parameters) by PSO and evaluating them with upper and lower bounds of the variable. The evaluation is managed by the information theory measures (entropy and mutual information) as the fitness function to generate membership function.

The process of generating membership function is divided into two phases:

- Phase 1 is the generation of three decision variables (used to parameterize membership function) by PSO.

- Phase 2 is the evaluation of the solution by total entropy function.

The two phases are iterated several times to reach algorithm satisfaction threshold or maximum number of iterations.

\subsection{Generating Random Decision Variables using PSO}

The procedure of generating membership function parameters (decision variables) by PSO is presented by the following steps:

Step 1: Generate initial swarm parameter

Step 2: Generate a Number of Decision Variables, let lower and upper values of data inputs is lower and upper bounds of decision variables (nvars)

Step 3: Evaluate the initial particles to get (pbest) and (gbest) by using fitness function (total entropy function)

Step 4: Evaluate updated particles to get new pbest and gbest by using entropy function

Step 5: if not maximum iteration, go to step 2

Step 6: Terminate PSO

Step 7: Build fuzzy model for the final population best particle (draw the membership function).

Step 8: End

The algorithm is illustrated in Figure 1: 


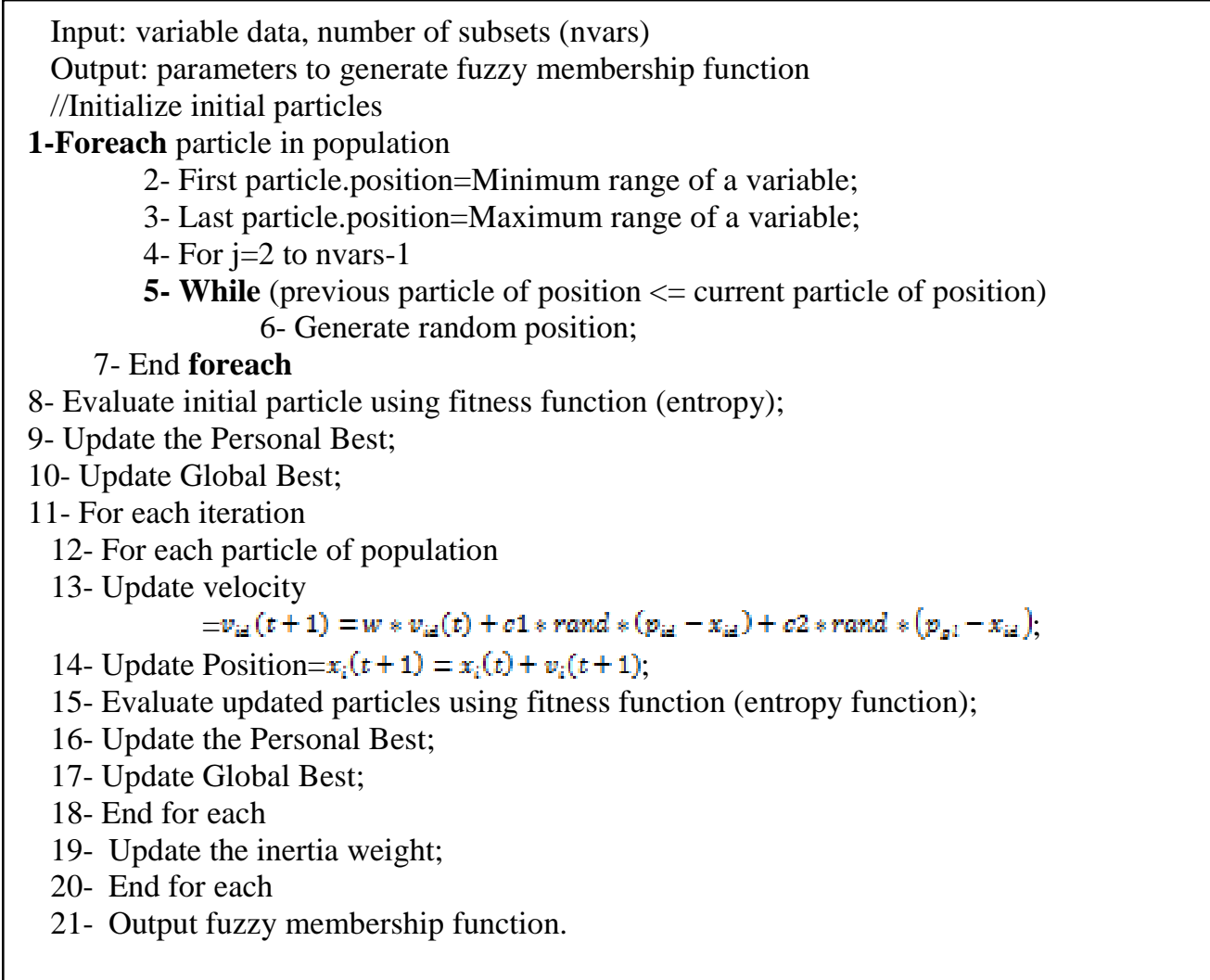

\section{Figure 2. Generating Unknown Decision Variables by PSO}

\subsection{Fitness Function}

The most important step in applying PSO is choosing an accurate fitness function. Fitness function [20] is utilized as a part of PSO, through iterations of the algorithm, to assess the nature of all the proposed solutions in the present population. The fitness function evaluates how good an individual solution in a population is.

There are numerous components that are imperative in managing a PSO inquiry to reach to the optimal solution. This paper use total entropy function as a fitness function in PSO algorithm. It is notable that entropy and information can be considered as measures of uncertainty of probability distribution. Uncertainty analysis of telecommunication input data will be evaluated by total entropy function to generate membership function [14][13].

The particles of PSO hold the membership function parameters of fuzzy variables. These particles should be evaluated to assure their ability to represent the data features efficiently. The total entropy function as an assessment method gives the validity to the entire system.

To calculate total entropy, it is very important to segment histogram into $\mathrm{m}$ joint segments that cause m-1 overlaps. This generates $\mathrm{m}$ participation capacities. Every joint segment addresses joint entropy. Whereas, every cover is displayed by mutual information demonstrating interdependency between two entropies. Equation No 6shows the total entropy function which is the fitness function. The total entropy (TE) is calculated by the set of parameters in the PSO particle and the variable data collected randomly from the available data space[6]:

$$
T E=\sum_{i=1}^{m} H(i)-\sum_{j=1}^{m-1} I\left(j_{j} j+1\right)
$$




\section{Where}

TE is the total entropy,

$\mathrm{H}(\mathrm{i})$ is the entropy of $\mathrm{i}^{\text {th }}$ partitions,

$I(j, j+1)$ is the mutual information between $j^{\text {th }}$ and $(j+1)^{\text {th }}$ partition

The PSO fitness procedure for evaluating particles is illustrated in the following steps:

Step 1: accept input data

Step 2: Divide 5 nvars into 3 ranges.

Step 3: Calculate frequency of bins in each range,

Step 4: Compute the probability for each range then compute entropy.

Step 5: Calculate joint entropy for each two adjacent subsets.

Step 6: Calculate mutual entropy (Equation No 5) for each two subsetthen calculate total entropy using equation No 6:

The algorithm of the fitness function evaluating is presented in figure NO 3:

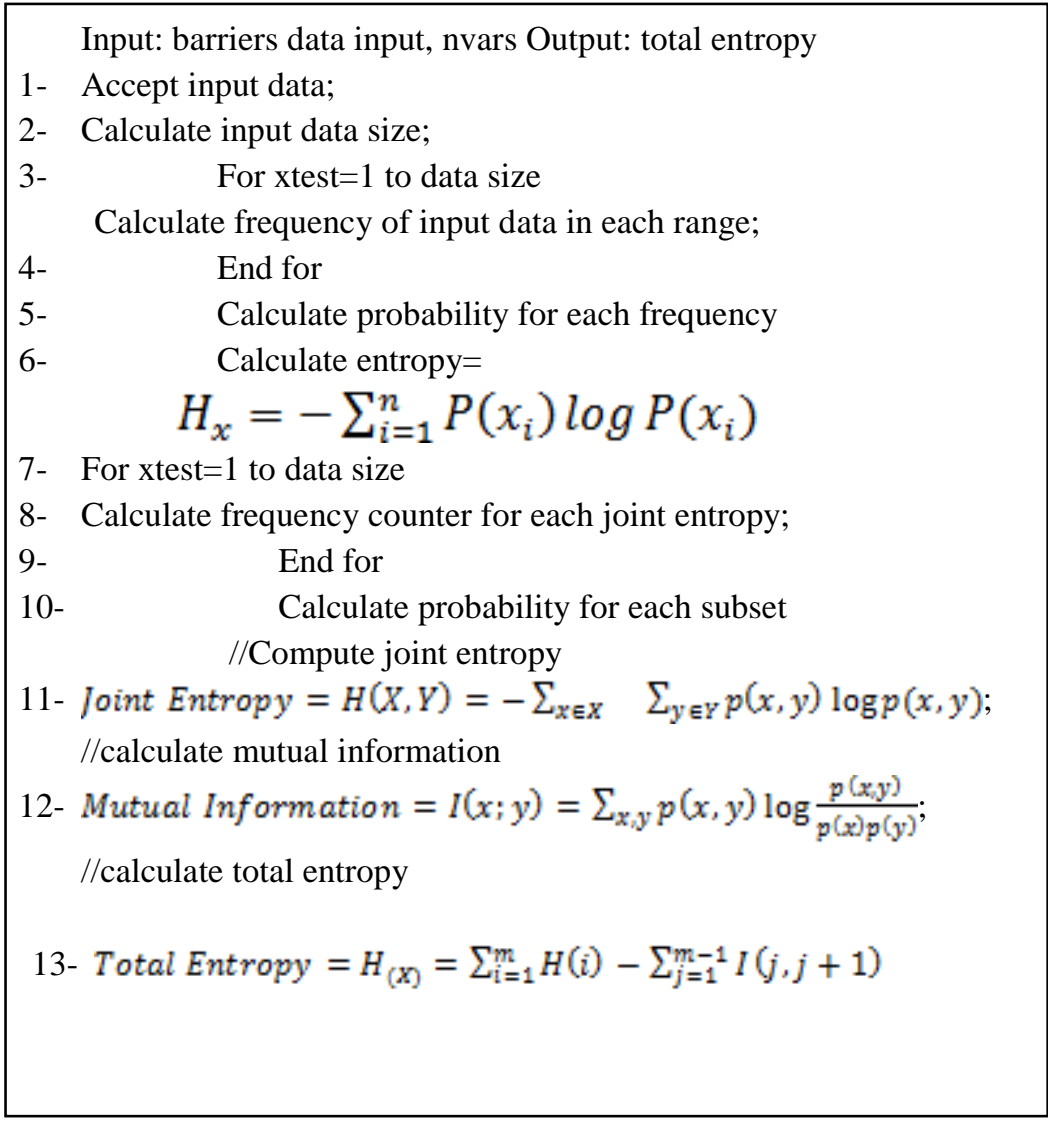

\section{Figure 3. Evaluate Particles Using Fitness Function (Total Entropy Function)}

Figure 4 illustrates the places of membership function parameters (decision variables) on a typical triangle membership function of a variable data space. Also the fitness function parameters (mutual information and entropy function) are shown 


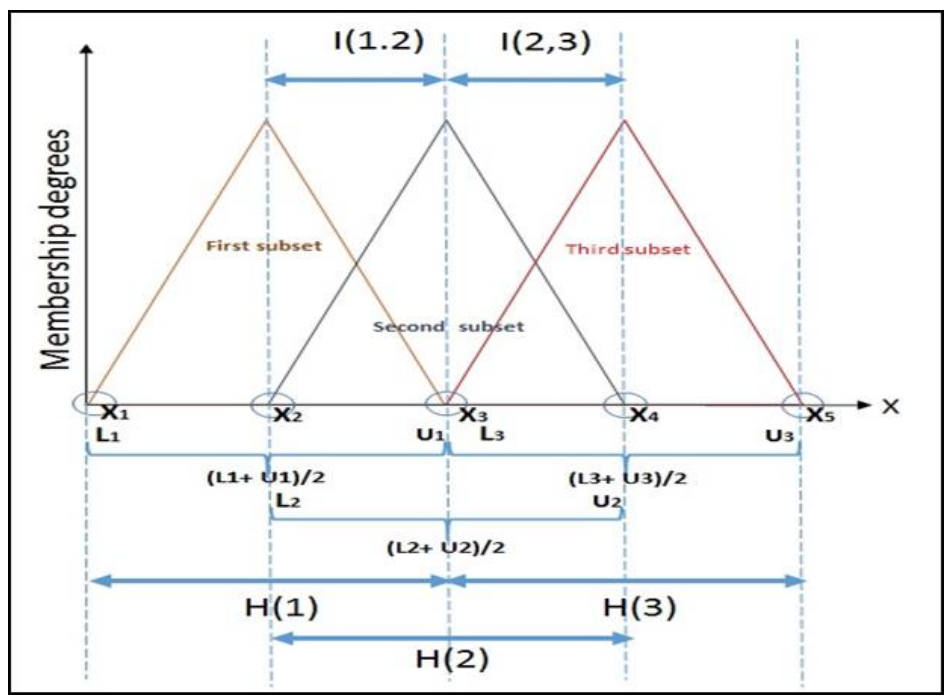

Figure 4. The Relationship between Total Entropy Co-Efficient and the Triangle Membership Function with 3 Subsets

According to Table 1 the PSO individual is designed to match decision points and constrains of the variable data space previewed in Figure 4.

Table 1. PSO Individual

\begin{tabular}{|c|c|c|c|c|}
\hline X1 & X2 & X3 & X4 & X5 \\
\hline $\begin{array}{c}\text { First Subset L1 } \\
\text { (Variable lower } \\
\text { bound ) }\end{array}$ & $\begin{array}{c}\text { Second } \\
\text { Subset L2 }\end{array}$ & $\begin{array}{c}\text { First Subset } \\
\text { U1 } \\
\text { Third Subset } \\
\text { L3 }\end{array}$ & $\begin{array}{c}\text { Second } \\
\text { Subset U2 }\end{array}$ & $\begin{array}{c}\text { Third Subset U3 } \\
\text { (Variable Upper } \\
\text { bound) }\end{array}$ \\
\hline
\end{tabular}

\section{Where}

$\mathrm{L}$ refers to the lower bound of the Triangle membership function of the subset,

$\mathrm{U}$ refers to the upper bound of the Triangle membership function of the subset,

The middle point is measured as $(\mathrm{L}+\mathrm{U}) / 2$. Further constrains are added to assure the integrity of the individual through calculations listed as:

1- The first decision variable must be constrained to the minimum value of the variable under the fuzzification process.

2- The last decision variable must be constrained to the maximum value of the variable under the fuzzification process.

3- All decision variables must be in an ascending order to avoid conflict membership degrees for variable values.

4- The upper bound of the first subset and the lower bound of the third subset are designed to be equalized. Hence a typical triangle membership function could be generated.

The flow chart of the PSO-TE integrated hybrid model is shown in Figure 6. 


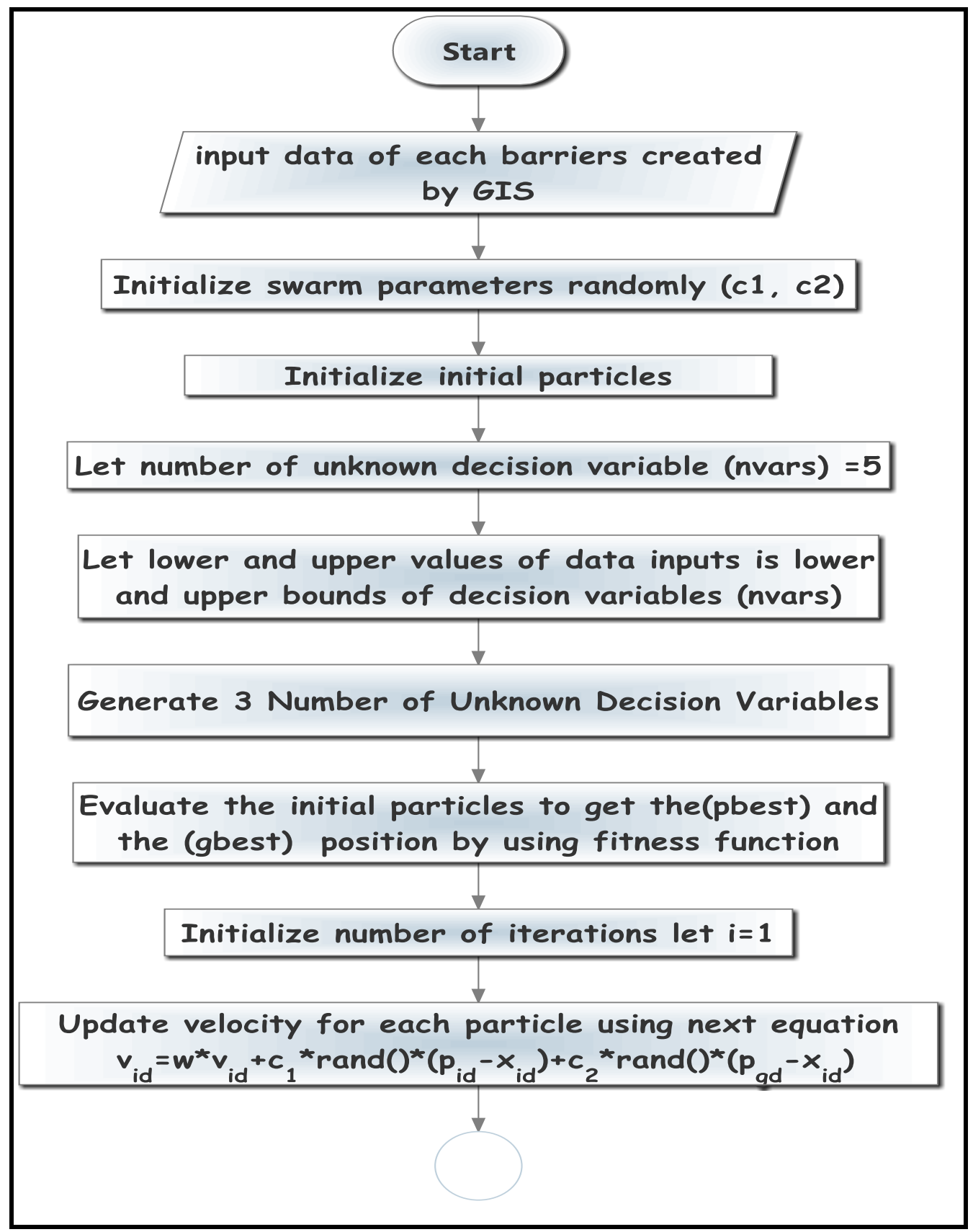

Figure 5. Flowchart of Particle Swarm Optimization for Designing Fuzzy Membership Function Part 1 


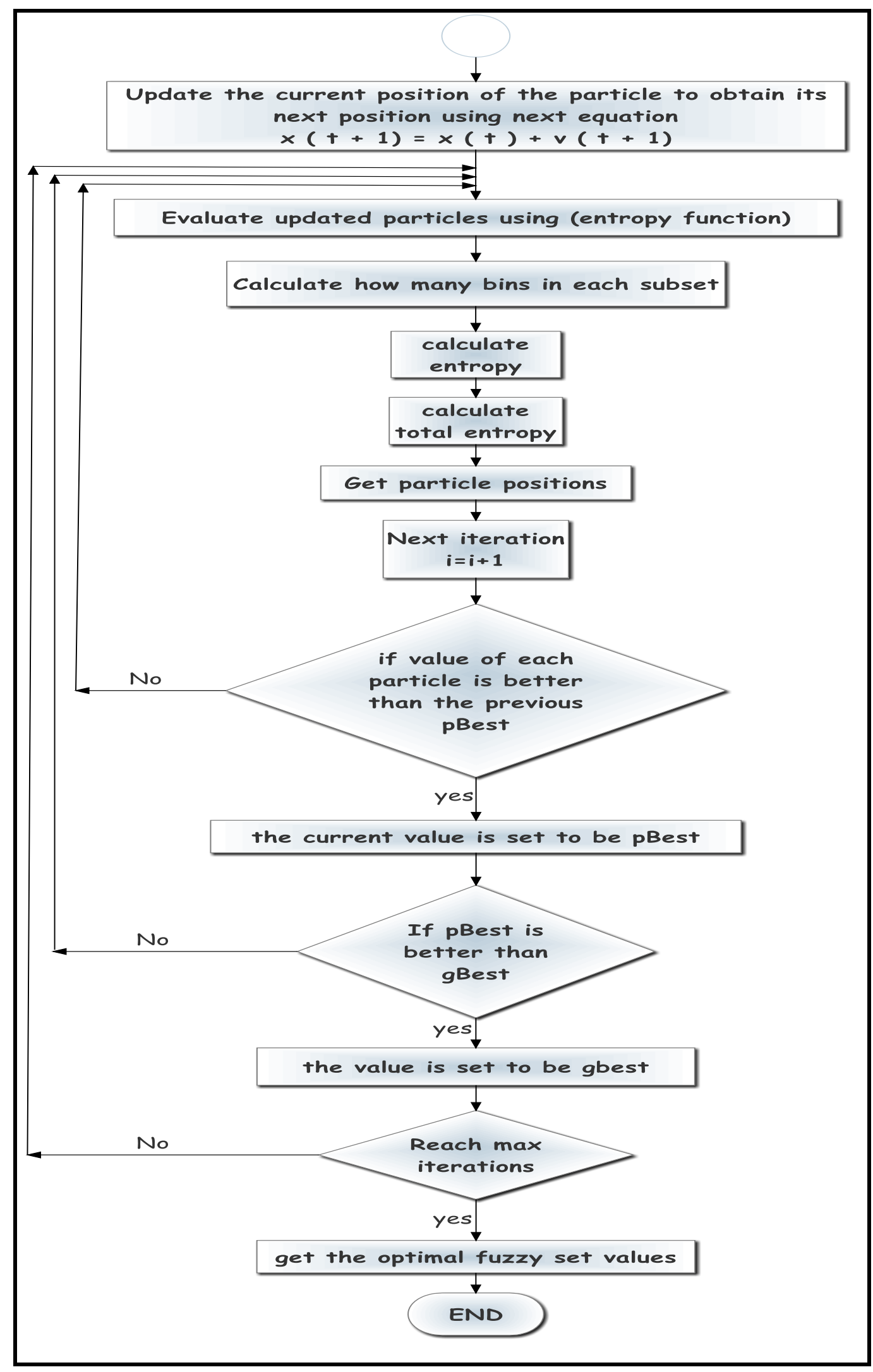

Figure 6. Flowchart of Particle Swarm Optimization for Designing Fuzzy Membership Function Part 2 


\section{Experimental Results}

\subsection{Telecommunication Data}

Geographic Information System (GIS) [11] is a computer system for capturing, storing, querying, analyzing and displaying geographic data. Spatial data is the most important part in GIS because of its ability of representing spatial location and attributive information.

This research use the geographic telecommunication data about access network infrastructure in telecom Egypt group which is represented by telecom Egypt network engineers using arcgis10.3 as an input data for the proposed algorithm. The researchers get all barriers of planning infrastructure from telecom Egypt planning standards. The following Figure 7 illustrate all these barriers.

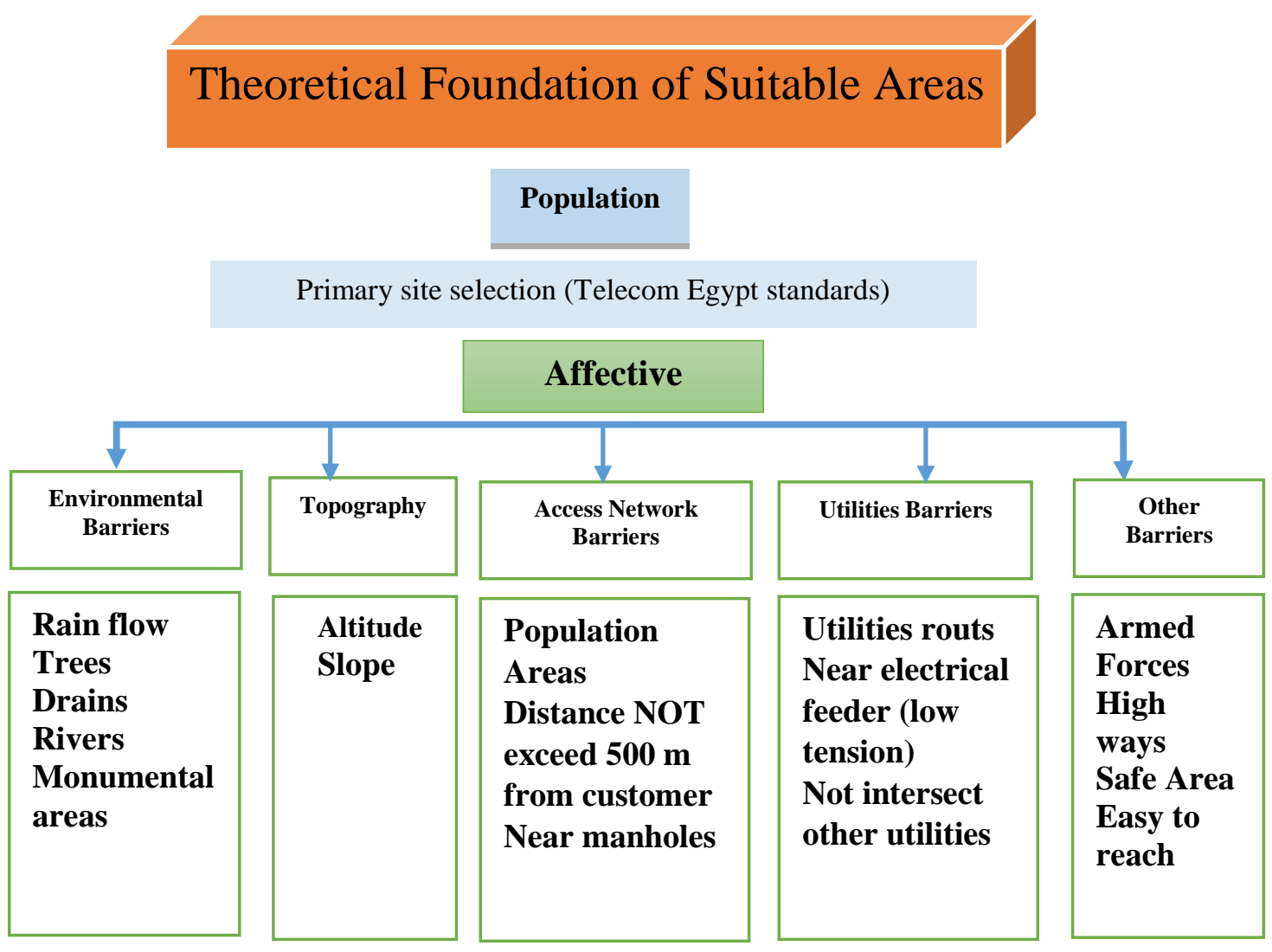

Figure 7. Theoretical Foundation of Suitable Areas

Figure 8 shows the area under study which is divided into small regions called Grid to analyze each region and determine existing barriers especially in the regions with existing MSAN (Multi Services Access Node). Also, future planned regions are taken within consideration. This research aims to preprocess the telecommunication data and transform it into fuzzy data. The telecommunication fuzzy data will be used later for determining the suitable location of MSAN in the region under study depending on pre_mentioned barriers. Figure8 shows a grid that covers the regions under the study. The researcher get the center of each grid and calculate the distance automatically between the center point of the grid and existing barrier individually such as drains, rivers and monumental areas. These barriers will be under the research study using arc toolbox. Each Grid has a unique number. Each grid may contain MSAN or not depending on existing access network. Table 1 shows a sample of barriers for some regions. 


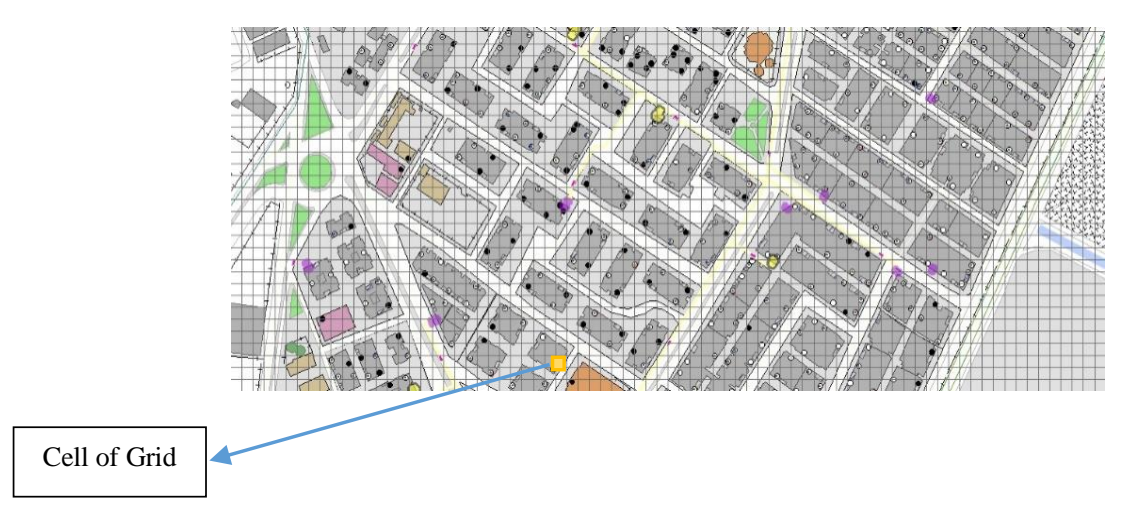

Figure 8. The Grid which Covers the Area Under Study

Table 2. Telecommunication Infrastructure Planning Barriers

\begin{tabular}{|c|c|c|c|c|}
\hline $\begin{array}{c}\text { Region } \\
\text { NO }\end{array}$ & Drains & Rivers & $\begin{array}{c}\text { Monumental } \\
\text { Areas }\end{array}$ & $\begin{array}{c}\text { Cabinet } \\
\text { Code }\end{array}$ \\
\hline 1 & 5 & 5 & 2 & $13-3-12-02$ \\
\hline 2 & 3.5 & 3 & 3.2 & $13-3-12-01$ \\
\hline 3 & null & 2.5 & 4.2 & $13-3-12-503$ \\
\hline 4 & 2.5 & 1.5 & 1.1 & ------ \\
\hline
\end{tabular}

Above table shows sample of barriers that determine whether these regions have environmental factors such as (Monumental areas, Rivers, Drains...etc) or not.

\subsection{Simulation Parameters}

The proposed PSO-TE (Particle Swarm Optimization- Total Entropy) is simulated by MATLAB r2015a software. The simulation is accomplished on an Intel Core i7-4510 U CPU processor, 6GB of RAM, and 1tera hard drive. The PSO parameters are set as: swarm size npop $=50$, maximum iteration MaxIt $=50$, learning factor $\mathrm{cl}=\mathrm{c} 2=2.0$, Inertia weight $\mathrm{w}=1$ and number of unknown variables nvars $=5$.

\subsection{Proposed Model}

The PSO-TE is an integrated hybrid model to generate fuzzy membership function for telecommunication data barriers (features). The membership function describes the degree of truth for the values in their corresponding subsets within the barriers. The PSO-TE benefits the PSO searching capabilities to find membership function parameters illustrated in section IV.B for the telecommunication barriers. The fitness function of the PSO is the maximization of total entropy function (entropy and mutual information) applied on the spatial input data of the barriers. Figure 5-b1, b2, b3 are examples of the membership function generated by the PSO-TE for telecommunication barriers namely (drains, trees and Monumental areas). The average time of the proposed integrated hybrid model is 2 sec. Figure 5-a1, a2 a3 shows the PSO-TE convergence rate for converting the same three telecommunication barriers. The algorithm converges quickly illustrating the ability of the proposed model to find best solutions within appropriate time. 


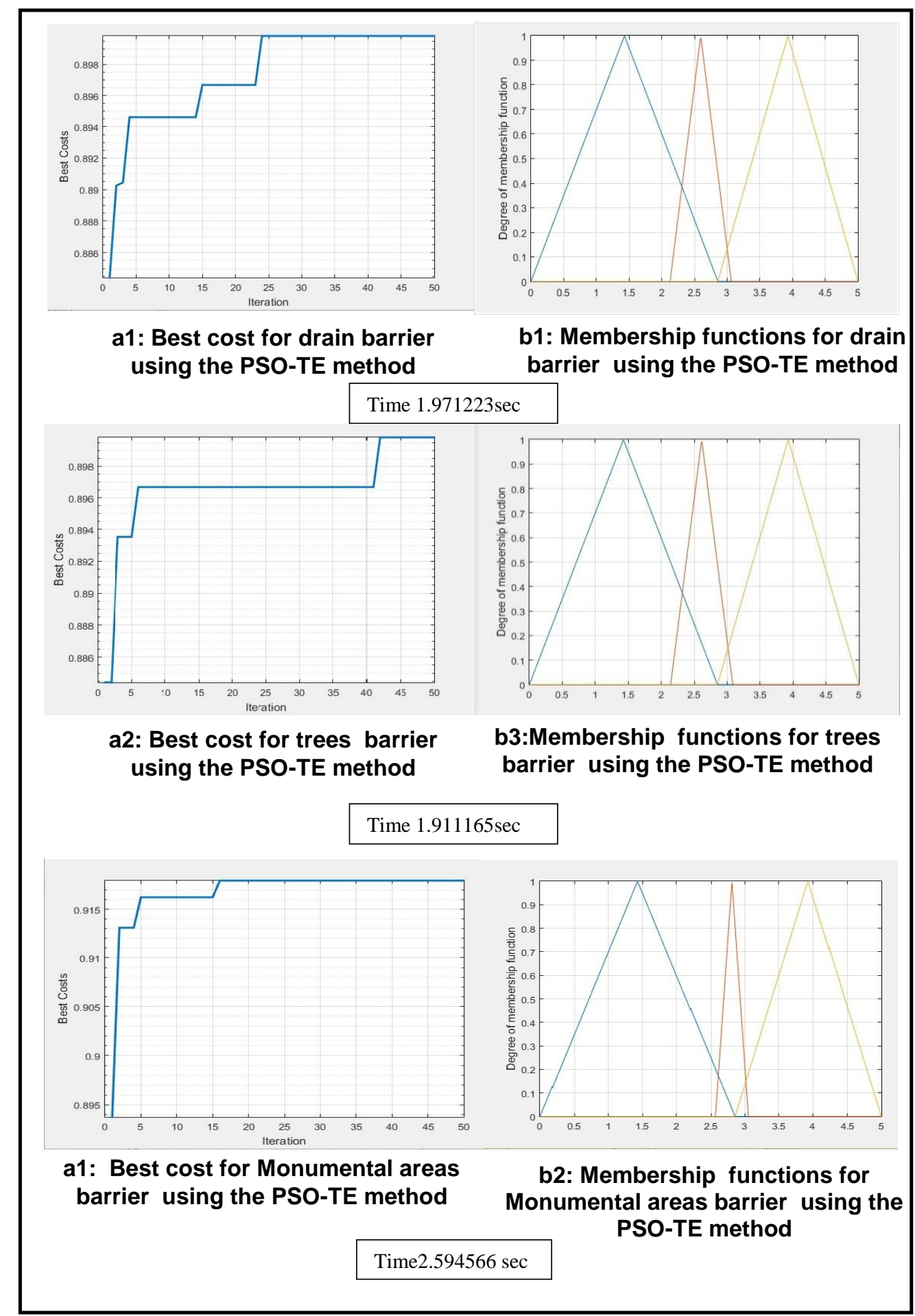

Figure 9. Membership Functions of the Spatial Input Data Using the PSO-TE Process 
Experiments were made to provide a comparison between the proposed hybrid model PSO-TE and fuzzy c_mean methods. The comparison of two methods based on their capability to generate a stable membership function effectively. The membership function of C-mean method and proposed hybrid model PSO-TE is experimented on two different random data sets taken from (Near electrical feeder and near manhole) barrier. Figures 10 $\& 12$ show the analogue membership function for the barrier generated by fuzzy C-mean. It's clear that the two pictures are different which means that the fuzzy C-mean algorithm cannot generate a stable membership function. Figures $11 \& 13$ represent the triangle membership functions generated by PSO-TE for the same random sets of the barrier. It seems that the PSO-TE produces a feasible membership functions for the telecommunication barriers.

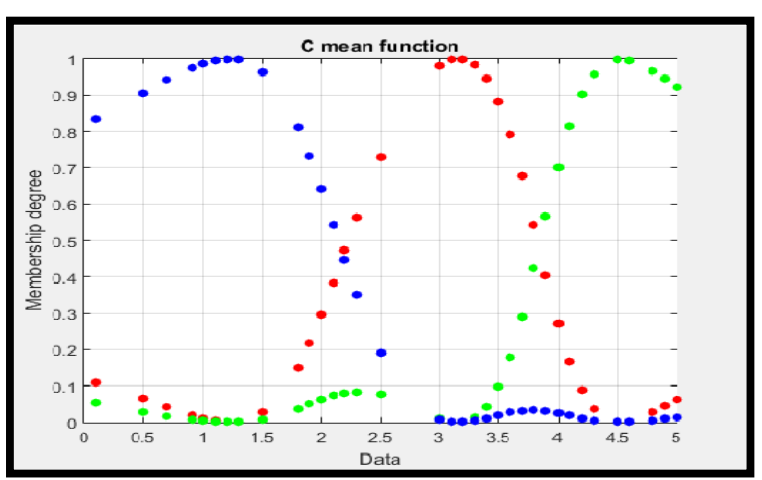

Figure 10. Membership function for Near Electrical Feeder Barrier Using Cmean Method

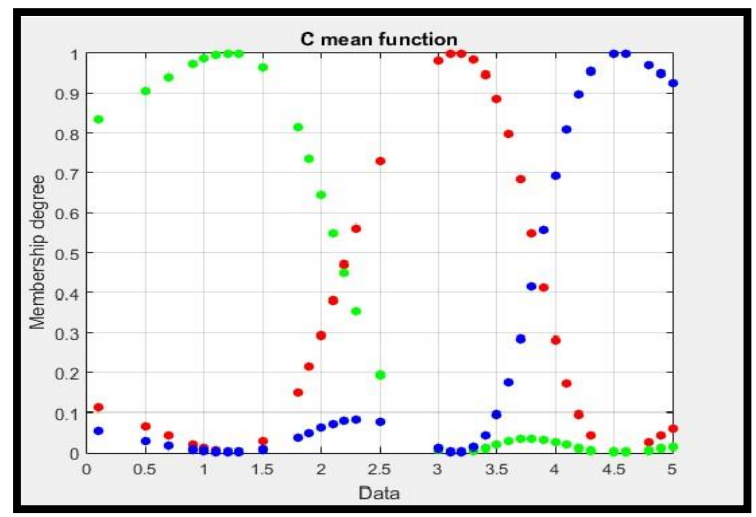

Figure 12. Membership Function for Manhole Barrier Using C-mean Method

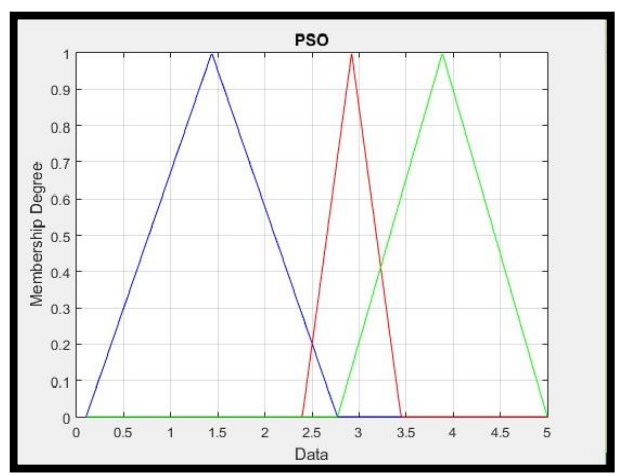

Figure 11. Membership function for Near Electrical Feeder Barrier Using PSO-TE Method

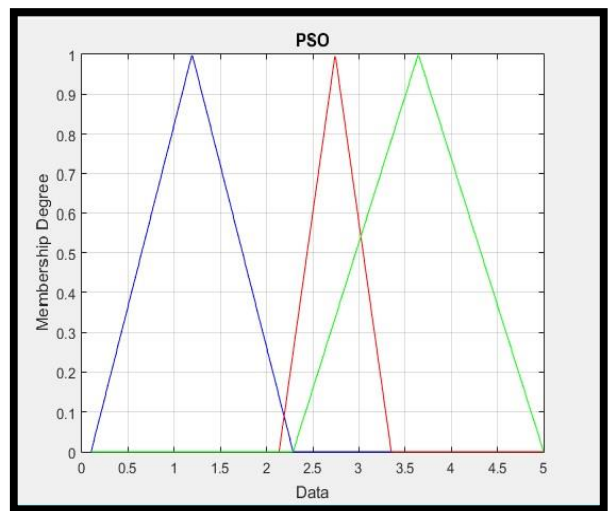

Figure 13. Membership Function for Manhole Barrier Using PSO-TE Method 


\section{Conclusion}

Telecommunication networks are essential in human lives nowadays. Therefore, there is an increasing need to analyze and manage telecommunication data carefully. The main obstacle is the amount of uncertainty and ambiguity found in the telecommunication data. Fuzzy systems are considered the official way of handling uncertainty away from the complexity of the probability calculations. The fuzzification is the first step in building a complete fuzzy system. This paper presents the ability of a proposed PSO-TE to generate membership function automatically for telecommunication network parameters. PSO-TE is an integrated hybrid model that deals with uncertainty GIS data and generates membership functions which help in making a complete fuzzy system for site selection. The model utilizes the PSO as a meta-heuristic search algorithm to find the best parameters for drawing a triangle membership function for each network barrier. The total entropy function works as the fitness function which evaluates the particles of the PSO. The total entropy is a feasible evaluation function as it represents the information conveyed within the barrier itself. The experimental results show a suitable triangle membership functions for the environmental barriers such as (drains, rivers and monumental areas). The convergence rate is presented showing the PSO-TE ability to find best solutions fast. The average time of the PSO-TE for the three experiments is 2 seconds. The telecommunication fuzzification process is an initial phase in decision making on fuzzy data and uncertainty analysis. Making fuzzy decisions on the fuzzy telecommunication data would be a suitable future point of research.

\section{References}

[1] A. Kalhora, B. N. Araabib, C. Lucas. "Generating flexible convex hyper-polygon validity regions via sigmoid-based membership functions in TS modelling." Elsevier -Applied Soft Computing, vol. 28 (2015), pp. 589-598.

[2] C. Wang . "A Study of Membership Functions on Mamdani-Type Fuzzy Inference System for Industrial Decision-Making." Lehigh Preserve, (2015), pp. 3-6.

[3] E. Hadavandi, H. Shavandi, A. Ghanbari, "Integration of genetic fuzzy systems and artificial neural networks for stock price forecasting." Elsevier B.V, (2010), pp. 800-808.

[4] F. Alikhademi, S. Zainudin, ed. "Generating of Derivative Membership Functions for Fuzzy Association Rule Mining by Particle Swarm Optimization", International Conference on Computational Science and Technology, (2014), pp. 1-6.

[5] G. Nieradka, B. Butkiewicz. "A Method for Automatic Membership Function Estimation Based on Fuzzy Measures." Springer-Verlag Berlin Heidelberg, (2007), pp. 451-460.

[6] G. Fang, N. M. Kwok and Q. Ha, " Automatic Fuzzy Membership Function Tuning Using the Particle Swarm Optimisation", IEEE-Pacific-Asia Workshop on Computational Intelligence and Industrial Application, (2008), pp. 324-328.

[7] H. M. Elragal, "Using Swarm Intelligence for Improving Accuracy of Fuzzy Classifiers." International Journal of Electrical, Computer, Energetic, Electronic and Communication Engineering, vol. 4, no. 8, (2010).

[8] ITU Telecom Network Planning. Telecom Network Planning for evolving Network Architectures, (2007).

[9] J. H. Chung, J. M. Pak, C. KiAhn, S. H. You, MyoTaegLim \& Moon Kyou Song. "Particle Filtering Approach to Membership Function Adjustment in Fuzzy Logic Systems", Elsevier -Neuro computing, (2016), pp. 1-20.

[10] K.K. Ang, C. Quek. "Supervised Pseudo Self-Evolving Cerebellar algorithm for generating fuzzy membership functions." Elsevier-Expert Systems with Applications, vol. 39, no. 3, (2012), pp. 22792287.

[11] K.-T. Chang, "Introduction Geographic Information Systems. Fifth Edition vols", North America: Mcgrow-Hill, (2010).

[12] K. E. Permana, S. Z. M. Hashim. "Fuzzy Membership Function Generation using Particle Swarm Optimization." Int. J. Open Problems Compt. Math, vol.3, no.1, (2010).

[13] L. S. Shafti, E. P'erez. "Fitness Function Comparison for GA-Based Feature Construction." SpringerVerlag Berlin Heidelberg, (2007), pp. 249-258.

[14] L. Zifa, Z. Jianhua, "Optimal Planning of Substation of Locating and Sizing Based on GIS and Adaptive Mutation PSO Algorithm", IEEE-International Conference on Power System Technology, (2006), pp. 15 . 
[15] M. Rahmat Widyanto, Rini Wijayanti. "Membership Function Modification in Fuzzy SVM using Combination of Distance Feature, Correlation, and Depth of Data", International Journal of Engineering \& Computer Science IJECS-IJENS, vol. 13, no. 5, (2013).

[16] M. Jian, S. Zengqi. "Mutual Information Is Copula Entropy", Tsinghua Science and Technology, vol. 6, no. 1, (2011), pp. 51-54.

[17] M. Makrehchi, O. Basir, M. Kamel. "Generation of Fuzzy Membership Function Using Information Theory Measures and Genetic Algorithm." Springer-Verlag Berlin Heidelberg, (2003), pp. 603-610.

[18] O. Adil, M. Ali, A. Y. Ali, B. S. Sumait, "Comparison between the Effects of Different Types of Membership Functions on Fuzzy Logic Controller Performance", International Journal of Emerging Engineering Research and Technology, vol. 3, no. 3, (2015).

[19] Q. Bai, "Analysis of Particle Swarm Optimization Algorithm." Computer and information science, vol. 3, no. 1, (2010).

[20] S.-H. Xu, J.-P. Liu, F.-H. Zhang, L. Wang , L.-J. Sun. "A Combination of A combination of Genetic Algorithm and Particle Swarm Optimization for Vehicle Routing Problem with Time Windows." Sensors, MDPI, (2015), pp. 21033-21053.

[21] T.W. Liao, "A Procedure for the Generation of Interval Type-2 Membership Functions from Data." Elsevier- Applied Soft Computing, (2016), pp. 1- 40.

[22] I. L. Wan, Y. S. Bih and Y. C. Chen, "A Hybrid Artificial Intelligence Sales-Forecasting System in the Convenience Store Industry", Wiley Periodicals, Inc, vol. 22, no. 3, (2012), pp. 188-196.

\section{Authors}

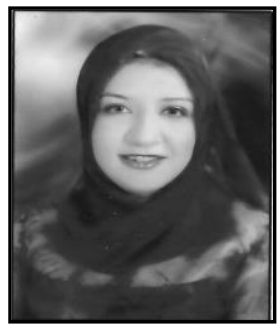

Walaa Elsanabary, she is Assistant Lecturer at Information system Department, Sadat Academy, Egypt. She received her M.S. degrees from the Department of Information system, Sadat Academy in 2012. She is also currently taking her Ph.D. at the Department of Information System, Mansoura University. Egypt.

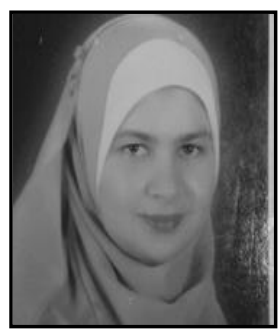

Mona Gamal, she is a Lecturer at Information system Department, Kafrelsheikh University, Egypt. She received her M.S. \& Ph.D. degrees from Information System Department, Faculty of Computers and Information Mansoura University, Egypt at 2010 and 2014 respectively

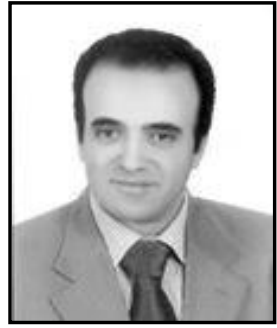

Ahmed Abou El-Fetouh, he is professor at Information system Department, Mansoura University, Egypt. He received Prof Degree in Information system Department from Faculty of Computers and Information Mansoura University, Egypt, in 2013 He is Vice Dean for Graduate Studies and Research in Egypt from 2013.

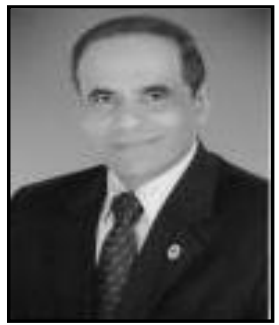

Nashaat Elkhameesy, he is Professor \& x Dean at the department of Information Systems, Sadat Academy for Management Sciences, Egypt. 